\title{
Comparative Voltammetric Behavior and Determination of Mephenesin in Pure Form, Pharmaceuticals and Biological Fluids at Pencil Graphite and Glassy Carbon Electrodes
}

\author{
Ehab El-Kady ${ }^{1}$, Hoda El-Qudaby², Marwa Omran ${ }^{2, *}$ \\ ${ }^{1}$ Pharmaceutical Chemistry Department, Faculty of Pharmacy, Cairo University, Cairo, Egypt \\ ${ }^{2}$ National Organization for Drug Control and Research (NODCAR), Giza, Egypt
}

Email address:

marwa251285@yahoo.com (M. Omran)

${ }^{*}$ Corresponding author

\section{To cite this article:}

Ehab El-Kady, Hoda El-Qudaby, Marwa Omran. Comparative Voltammetric Behavior and Determination of Mephenesin in Pure Form, Pharmaceuticals and Biological Fluids at Pencil Graphite and Glassy Carbon Electrodes. Science Journal of Analytical Chemistry.

Vol. 7, No. 2, 2019, pp. 32-41. doi: 10.11648/j.sjac.20190702.11

Received: February 28, 2019; Accepted: April 3, 2019; Published: May 7, 2019

\begin{abstract}
New voltammetric methods are introduced for the determination of a skeletal muscle relaxant namely; mephenesin (Mep) in its pure form with the application in the pharmaceutical preparation and biological fluids. Three voltammetric methods namely; cyclic voltammetry $(\mathrm{CV})$, differential pulse voltammetry (DPV) and square wave voltammetry (SWV) using pencil graphite electrode (PGE) and glassy carbon electrode (GCE) were used. Voltammetric behavior of Mep was investigated in Britton Robinson (BR) buffer as electrolyte in $\mathrm{pH}$ range 2-10. CV produced one irreversible anodic peak revealing oxidation reaction in $\mathrm{pH} 6$ as maximum $\mathrm{pH}$ value at 1.33 and $1.36 \mathrm{~V}$ using PGE and GCE, respectively. At PGE, DPV and SWV were investigated and linearity ranges were $18.02-119.07$ and $18.02-70.0 \mu \mathrm{g} / \mathrm{mL}$ with correlation coefficient 0.9997 and 0.9995 , LOD values were found to be 0.113 and $0.059 \mu \mathrm{g} / \mathrm{mL}$, respectively. At GCE, linearity ranges were $9.05-$ 44.39 and $4.54-65.78 \mu \mathrm{g} / \mathrm{mL}$ with correlation coefficient 0.9996 and 0.9999 , LOD values were 0.553 and $0.865 \mu \mathrm{g} / \mathrm{mL}$ at DPV and SWV, respectively. The proposed methods were applied with good recovery in pharmaceutical preparation, human mother milk and urine. They were validated and revealed accurate and precise results.
\end{abstract}

Keywords: Mephenesin, Pharmaceuticals, Biological Fluids, Glassy Carbon Electrode, Pencil Graphite Electrode, Voltammetry

\section{Introduction}

Mephenesin, 3-(2-methylphenoxy)propane-1,2-diol (Mep) (Figure 1), is a centrally acting muscle relaxant drug $[1,2]$ that can be used as an antidote for strychnine poisoning and a topical analgesic. However, it presents with the major drawbacks of having a short duration of action and much greater effect on the spinal cord than the brain, resulting in pronounced respiratory depression at clinical doses and therefore a very low therapeutic index. Mep is especially dangerous and potentially fatal in combination with alcohol and other depressants. Therefore, the development of new and simple analytical methods for rapid detection and quantification of Mep is of great importance that helps in earlier detection to prevent its side effects and intoxication.

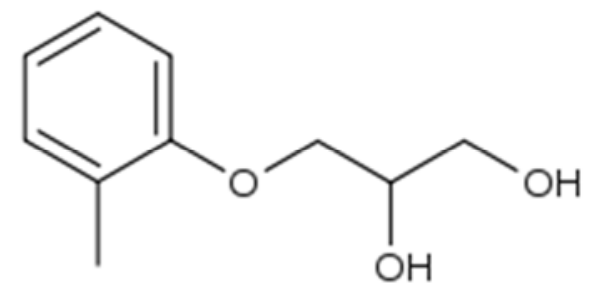

Figure 1. Chemical structure of Mephenesin.

Literature survey shows that several methods have been used to determine Mep including high-performance liquid chromatography separation with fluorimetric detection [3], Stability indicating HPLC method for simultaneous determination of mephenesin and diclofenac diethylamine 
with UV detection [4], spectrophotometric determination of Mep and guaifenesin in bulk and dosage forms that was based on the oxidation of them with sodium periodate to liberate formaldehyde which reacts insitu with acetylacetone in presence of ammonium acetate to give a yellow colored product [5], spectrophotometric determination of Mep and the principle metabolite, 3-(o-tolyloxy)-lactic acid, that was accomplished by the ether extraction of Mep from plasma and urine at alkaline $\mathrm{pH}$ followed by the extraction of the metabolite at $\mathrm{pH} 3$ [6] and spectrofluorometry [7]. These reported techniques are slow, sophisticated, requiring expensive instrumentations, elaborated and tedious extraction

Thus, the development of new voltammetric methods like $\mathrm{CV}$, DPV and SWV proved to be more simple, rapid, selective, sensitive, inexpensive with the advantages of no need for derivatization, less sensitivity to matrix effects than other analytical methods and capable of determining the drug in pure form and biological fluids directly. Additionally, application of electrochemistry includes the determination of the electrode mechanism and redox properties of the drug under study that give insights into their metabolic fates, invivo redox processes or pharmacological activity and great importance serving clinical investigations by introducing preliminary vision for pharmacokinetic studies [8-14].

The aim of this work is the development of new voltammetric methods for direct determination of Mep in pure form, pharmaceutical dosage form and application in spiked human mother milk and urine samples to introduce a good reference for the rapid detection and quantification of it in order to prevent its side effects and intoxication as it is excreted by kidney [2] and reported to be contraindicated during lactation [15]. Mother milk was used for doublet aims; as it is an alternative to serum to be used without centrifugation nor sedimentation so, save time, equipment and effort, moreover this application helps for earlier detection to prevent side effects in lactating mothers, therefore, we can introduce preliminary study on the pharmacokinetics on all patients specially lactating mothers. Furthermore, PGE and GCE provide the needed advantages as simplicity, cheapness, availability, being solid and environmental friendship over the reported methods for the determination of Mep, PGE provides more advantages to be disposable, cheaper and easier to be used [16-22].

\section{Experimental}

\subsection{Instrumental and Experimental Set-up}

Voltammetric measurements were obtained using the electrochemical analyzer Computrace system with 797VA Computrace software (1.0) from Metrohm, Switzerland. A three-electrode cell was employed. The working electrodes were PGE, a HB Rotring hi polymer with a diameter of 0.7 $\mathrm{mm}$ identifiable with (S0312690, R505708N). Electrical contact with the pencil electrode was achieved by soldering a copper wire to the metallic part of the apparatus fixing the leads, $8 \mathrm{~mm}$ of the electrode was exposed in contact with the electrolyte to be measured [23] and GCE, a mini glassy carbon disk electrode of the active zone: 2.8mm, for ELCD 641/656. To improve the sensitivity and resolution of the voltammetric peaks, the GCE was polished manually with alumina slurry on a smooth polishing cloth prior to each electrochemical measurement. Then, it was thoroughly rinsed with methanol and doubled distilled water, sonicated for 10 minutes and dried with a piece of paper [24]. A reference electrode, $\mathrm{Ag} / \mathrm{AgCl}(3$ mol. $\mathrm{L}^{-1} \mathrm{KCl}$ ) was used and a platinum wire as a counter electrode. A Mettler balance (Toledo-AB104) was used for weighing the solid materials, U.S.A. A micropipette (Eppendorf- multipette plus) was used throughout the present experimental work, German. The $\mathrm{pH}$ measurements were performed using Jenway3330 Research pH meter, U.K. Used deionized water throughout the present study was supplied from a burette still plus deionized connected to a HamiltonAqua-Metric deionized water system, U.K. All the experiments were performed at room temperature.

\subsection{Chemicals and Reagents}

Mep was supplied from Zad company, potency was certified to be $99.67 \%$. Decontractyl ${ }^{\circledR} 500 \mathrm{mg}$ tablets (each labeled to contain $500 \mathrm{mg}$ mephenesin- Sanofi Aventis Pharmaceutical Company, France). A stock solution of 1820 $\mu \mathrm{g} / \mathrm{mL}\left(10^{-2} \mathrm{M}\right) \mathrm{Mep}$ raw material was freshly prepared by dissolving the weighed amount of $182 \mathrm{mg}$ in $100 \mathrm{~mL}$ deionized water and stored in refrigerator $4{ }^{\circ} \mathrm{C}$. A similar solution of the dosage form was prepared by crushing ten Decontractyl ${ }^{\circledR}$ tablets and finely powdering using a mortar and pestle. A weighed portion of the powder equivalent to $182 \mathrm{mg}$ of Mep was transferred to a $100 \mathrm{~mL}$ volumetric calibrated flask, dissolved in $50 \mathrm{~mL}$ distilled water by sonication for $5 \mathrm{~min}$ to achieve complete dissolution, complete to the mark with the same solvent, filtered through a filter paper and the first $10 \mathrm{~mL}$ of the filtrate was discarded. Britton- Robinson (B-R buffer) of concentration 0.04 M was prepared by mixing acetic, boric and phosphoric acids [25, 26]. Appropriate amounts of $0.2 \mathrm{M} \mathrm{NaOH}$ were added to obtain the desired $\mathrm{pH}(2.0$ to 10.0$)$. All solutions were prepared from analytical grade chemicals and deionized water. All materials and reagents were used as received without further purification.

\subsection{Recommended Experimental Procedures}

\subsubsection{Assay of Pure Form}

In the electrochemical measurements, voltammetric analyses were performed in $10 \mathrm{~mL}$ of B-R buffer solution while using PGE and $20 \mathrm{~mL}$ with GCE. Appropriate aliquots of the drug-solution of Mep were introduced into the electrolytic cell successively and the calibration curves of Mep using DPV and SWV were constructed by plotting the peak current $\mathrm{I}(\mu \mathrm{A})$ against drug concentration $(\mu \mathrm{g} / \mathrm{mL})$.

\subsubsection{Application of the Proposed DPV and SWV Methods for the Determination of Mep in Pharmaceutical Dosage Form}

The methods (DPV at PGE) and (SWV at GCE) were 
performed using the sample preparation of the dosage form with applying the standard addition technique.

The selected methods were chosen to give some while wider ranges than others to allow the application of standard addition technique.

\subsubsection{Application to Human Mother Milk and Urine Samples}

Direct application to human mother milk and urine without any sample pretreatment like precipitation was carried out using SWV. Milk and urine samples were supplied from healthy volunteers from the team work, allowed to settle for about 30 minutes and then used for the experiments one at a time. $10.0 \%$ of B-R buffer was replaced by the biological fluid in the voltammetric cell then Mep solution was added with concentrations of applicable range subsequently then analyzed according to the recommended in the general analytical procedure.

\section{Results and Discussion}

\subsection{Electrochemical Oxidation of Mep}

The voltammetric behavior of Mep was studied at PGE and GCE using $0.04 \mathrm{M}$ B-R buffer solution as supporting electrolyte investigating different chemical and electrochemical parameters.

Applying cyclic voltammetry; Mep showed one irreversible anodic peak at 1.33 and $1.36 \mathrm{~V}$ at PGE and GCE, respsctively, The major oxidation product of mephenesin has been identified as 3-(2-methylphenoxy)-2-ketone-1-propanol, may be owing to the oxidation of hydroxyl group to ketone group as depicted by the cyclic voltammogram given in (Figure 2) and proposed mechanism in (Figure 3) [27].

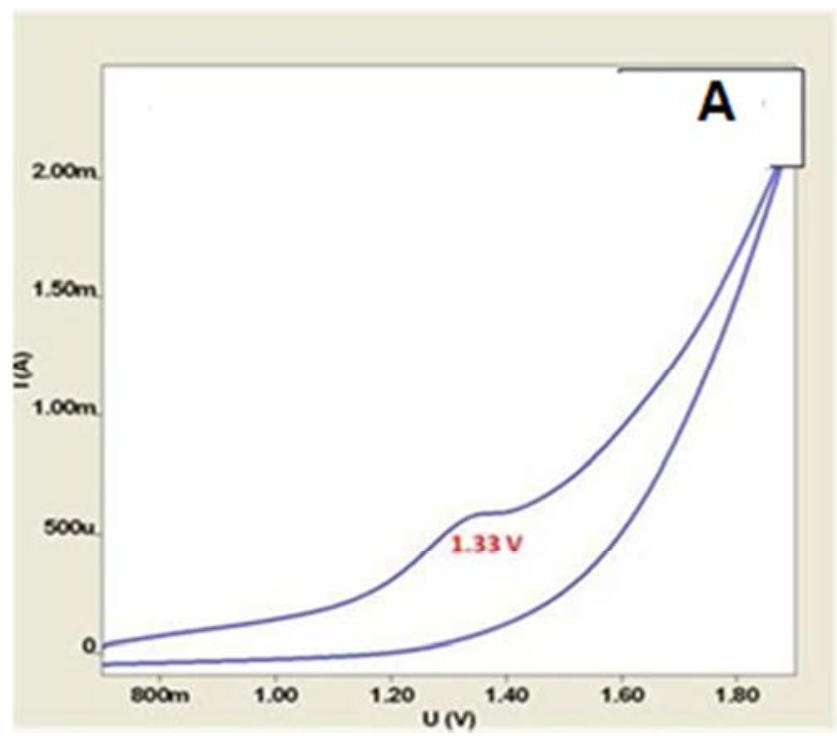

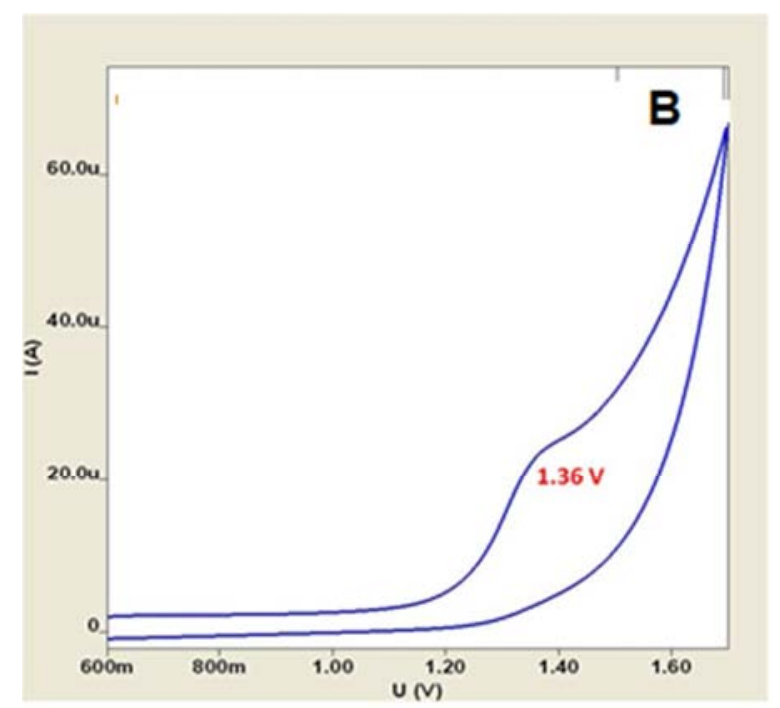

Figure 2. Cyclic voltammograms of $44.39 \mu \mathrm{g} / \mathrm{mL}$ Mep in $0.04 \mathrm{M}$ BR buffer pH 6.0 applying scan rate $\left(0.18 \mathrm{mV} . \mathrm{s}^{-1}\right)$ at (A) PGE and (B) GCE.

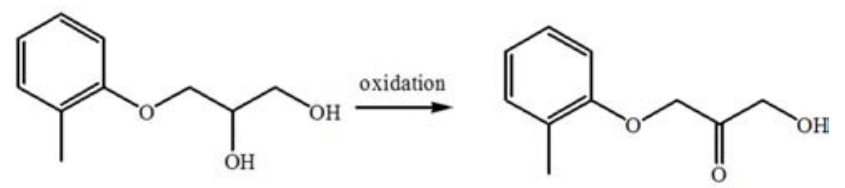

Figure 3. Proposed mechanism of the electrochemical reaction of Mep in $0.04 M$ BR buffer $p H 6.0$ at (A) PGE and (B) GCE.

\subsection{Optimization of Experimental Conditions}

\subsubsection{Effect of pH of the Supporting Electrolyte}

The $\mathrm{pH}$ of the electrolyte medium is one of the variables that commonly and strongly affects the shape of the voltammogram, therefore it was important to investigate the effect of the $\mathrm{pH}$ on the electrochemical behavior of the drug. The influence of $\mathrm{pH}$ on the peak current was examined using SWV for Mep solution in $0.04 \mathrm{M}$ B-R buffer. Experiments at $\mathrm{pH}$ values (2.0 to 10.0 ) were carried out to assess the $\mathrm{pH}$ impact on the monitored electro-analysis signal at both electrodes. The observed signals were $\mathrm{pH}$-dependent, there were changes of peak current with different $\mathrm{pH}$, the maximum peak current was obtained at $\mathrm{pH} 6$ when the most sharp and symmetrical peaks in the assay experiments were obtained.

Hence it was used as an optimum $\mathrm{pH}$ value throughout the whole study (Figure $4 *$ ).

The effect of solution $\mathrm{pH}$ on peak potential of Mep at both electrodes was also investigated. Figure (4 ) shows the change of the peak potential with different $\mathrm{pH}$ values where the oxidation peak potential of Mep shifts linearly to reduced values with the increase of $\mathrm{pH}$ of the medium, denoting that protons may be involved in the electrode reaction process [29] and linear correlations between $\mathrm{pH}$ and peak potential Ep (V) was found in equations (1\&2) as follow:

$$
\begin{aligned}
& \text { At PGE: } \mathrm{Ep}(\mathrm{V})=-0.0119 \mathrm{pH}+1.3355 \mathrm{R}^{2}=0.9921 \\
& \text { At GCE: } \mathrm{Ep}(\mathrm{V})=-0.0077 \mathrm{pH}+1.3654 \mathrm{R}^{2}=0.9903
\end{aligned}
$$



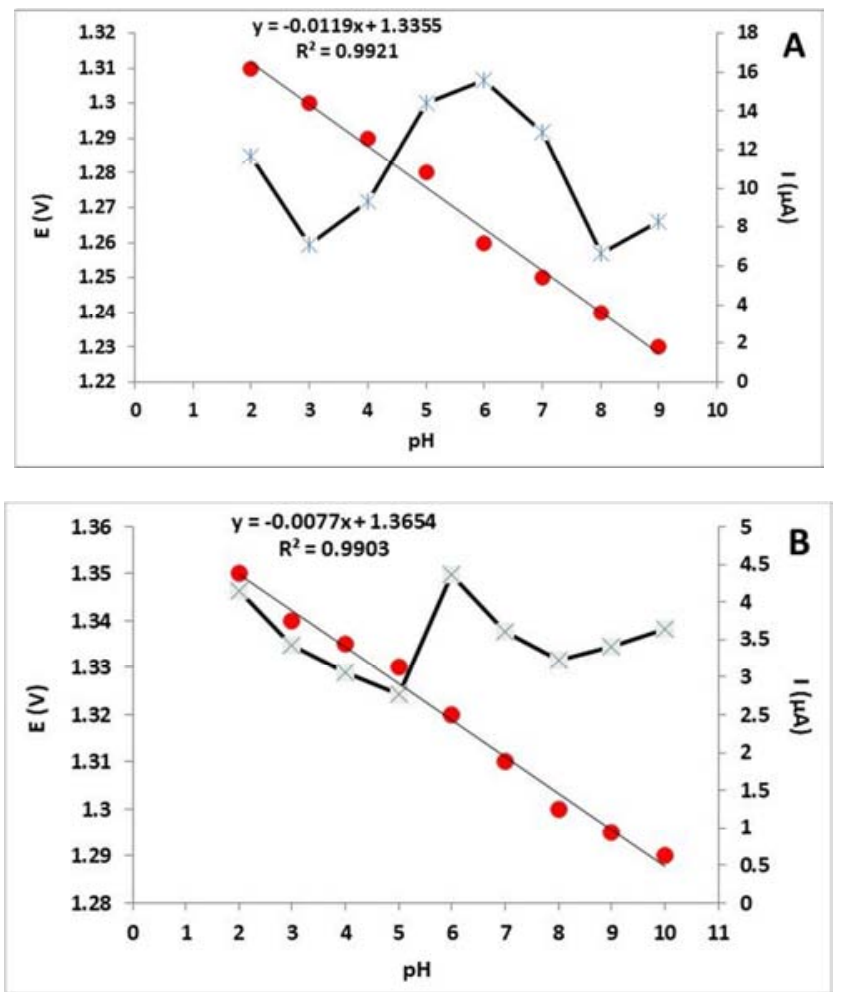

Figure 4. Effect of $p H$ on the peak current $(I) *$ and potential (E) for Mep in $0.04 M$ BR buffer at (A) PGE and (B) GCE.

\subsubsection{The effect of Voltammetric Parameters}

\section{Effect of Scan Rate}

Scan rate studies were carried out to assess whether the electrochemical process was under diffusion or adsorption controlled process using cyclic voltammetry, applying scan rate from $\left(0.02-0.18{\mathrm{~V} . \mathrm{s}^{-1}}^{-1}\right)$ at PGE and $\left(0.08-0.3 \mathrm{~V} . \mathrm{s}^{-1}\right)$ at GCE that is ensured through plotting peak current I $(\mu \mathrm{A})$ versus scan rate $\left({\mathrm{V} . \mathrm{s}^{-1}}^{-}\right)$(Figure 5), which gave linear relation with equations $(3 \& 4)$ :

$$
\begin{aligned}
& \text { At PGE: } \mathrm{I}(\mu \mathrm{A})=722.33 \mathrm{v}+6.7778 \mathrm{R}^{2}=0.9928 \\
& \text { At GCE: } \mathrm{I}(\mu \mathrm{A})=14.815 \mathrm{v}+0.6819 \mathrm{R}^{2}=0.9972
\end{aligned}
$$

Plotting log current I ( $\mu \mathrm{A})$ versus log scan rate gave a straight line with a slope value (0.9693) in equation (5) at PGE near the theoretical value of 1.0 ensuring adsorptioncontrolled electrode process [30] and (0.7917) in equation (6) at GCE between 0.5 and 1.0 revealing adsorption controlled electrode process with some diffusion that could be related to the contribution of thin layer electrolysis [31, 32, 33]:

At PGE: $\log \mathrm{I}(\mu \mathrm{A})=0.9693 \log \mathrm{v}+2.8655 \mathrm{R}^{2}=0.9973$

At GCE: $\log \mathrm{I}(\mu \mathrm{A})=0.7917 \log \mathrm{v}+1.1198 \mathrm{R}^{2}=0.9996(6)$

The peak potential $\mathrm{E}(\mathrm{V})$ shifted to more positive values on increasing the scan rate at PGE, which confirms the irreversibility of the oxidation process, that is shown through the linear relation between peak potential versus log scan rate at PGE which can be expressed in equation (7):

$$
\mathrm{E}=0.1472 \log \mathrm{v}+1.5158 \mathrm{R}^{2}=0.9946
$$

The peak potential didn't shift at GCE.

While plotting log current VS log scan rate, the slope value closes to the theoretical value of 0.5 refers to an ideal reaction to the diffusion process, when it closes to the theoretical value of 1.0 , it refers to an ideal reaction to the adsorption process $[34,35,36]$. However, the intermediate value of the slope is observed, suggesting a mixed adsorption-diffusion controlled process [20]. The peak potential shifted to more positive values on increasing the scan rate, which confirms the irreversibility of the oxidation process [30].
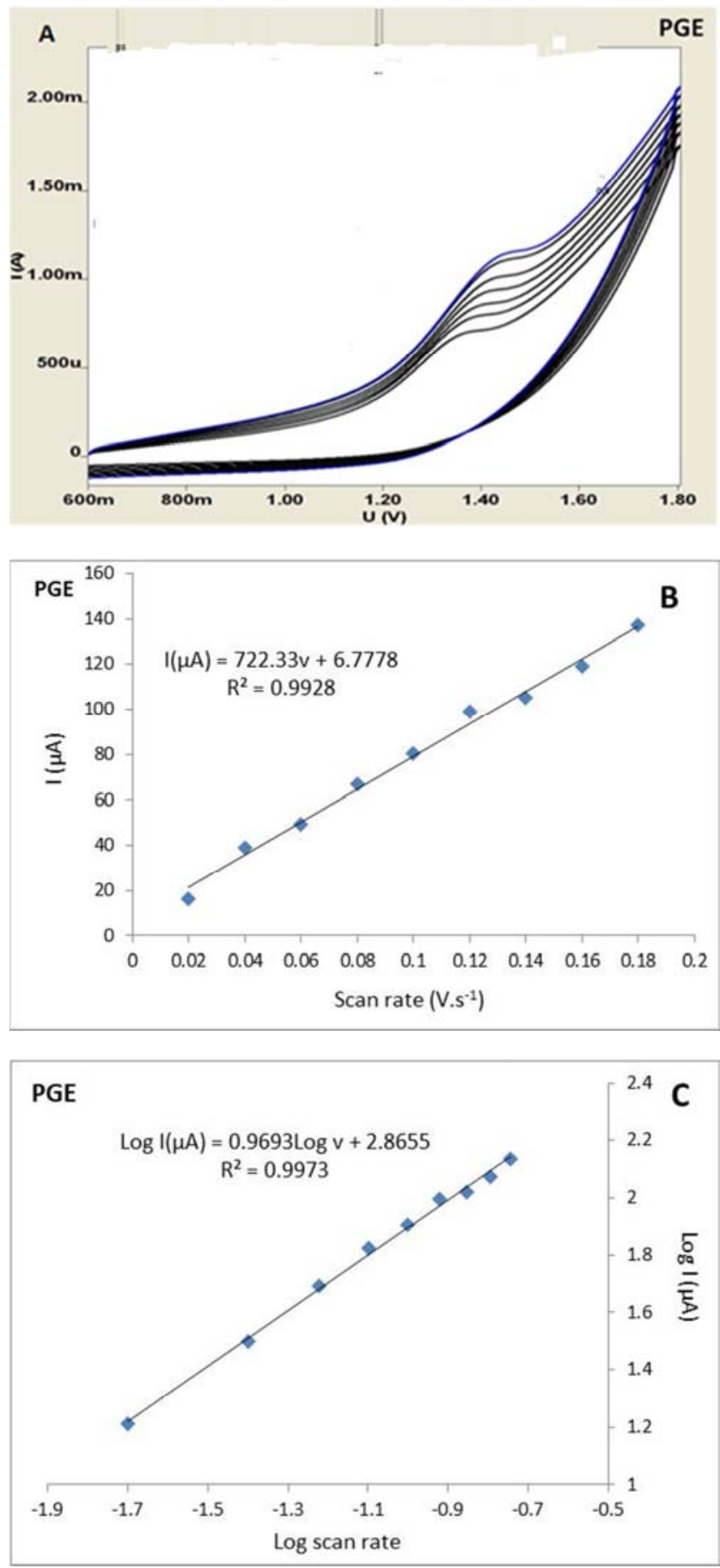

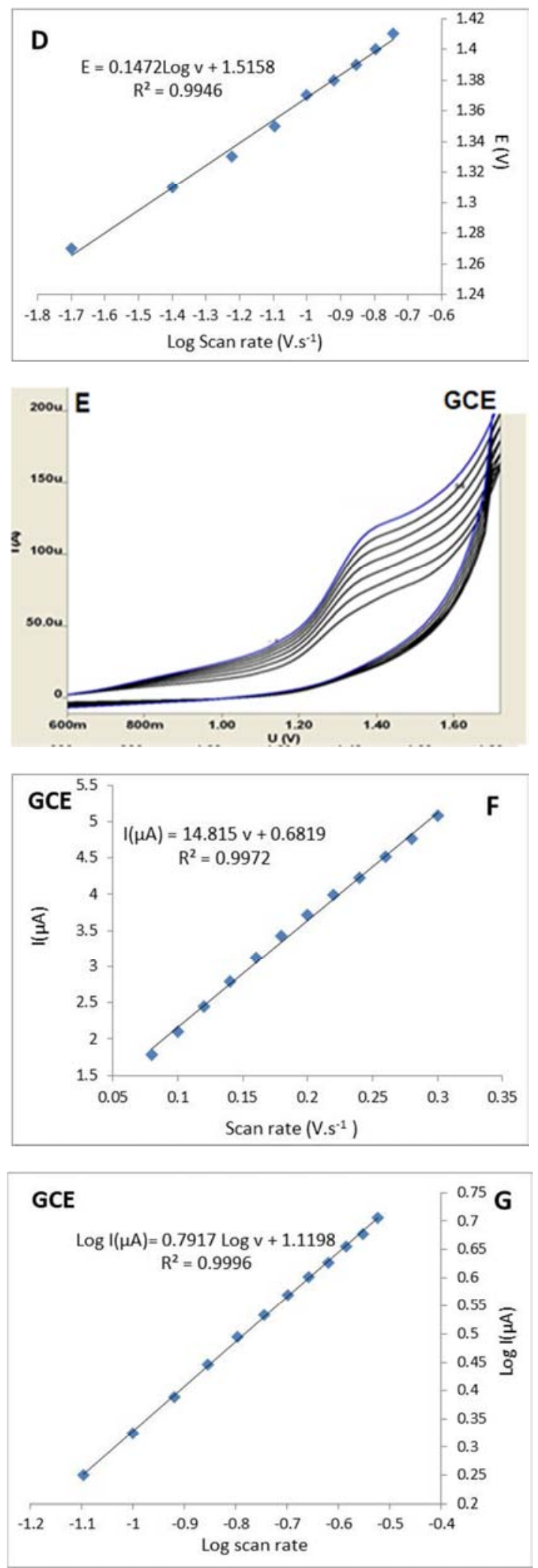

Figure 5. (A) Cyclic voltammograms of $44.39 \mu \mathrm{g} / \mathrm{mL}$ of Mep in $0.04 \mathrm{MBR}$ buffer ( $p H$ 6.0) at PGE, (B) The plot of peak current I ( $\mu A)$ of Mep versus scan rate $\left(V . s^{-1}\right)$ at $P G E$, (C) The plot of log peak current of Mep versus log scan rate at $P G E,(D)$ The plot of peak potential $E(V)$ versus log scan rate at PGE, (E) Cyclic voltammograms of $70.0 \mu \mathrm{g} / \mathrm{mL}$ of Mep in $0.04 \mathrm{M} \mathrm{BR}$ buffer ( $p H$ 6.0) at GCE, (F) The plot of peak current I $(\mu A)$ of Mep versus scan rate $\left(V . s^{-1}\right)$ at $G C E$ and $(G)$ The plot of log peak current of Mep versus $\log$ scan rate at $G C E$.

\section{Effect of deposition potential}

The effect of deposition potential as a function of the peak current was evaluated for an appropriate amount of Mep solution introduced to the electrolytic cell showing 0.2 and 0.4 as the best values to be applied in the whole experiments for PGE and GCE as shown in (Figure 6), respectively. The adsorptive peak current at the electrodes' surfaces appear to be dependent on the deposition potential.

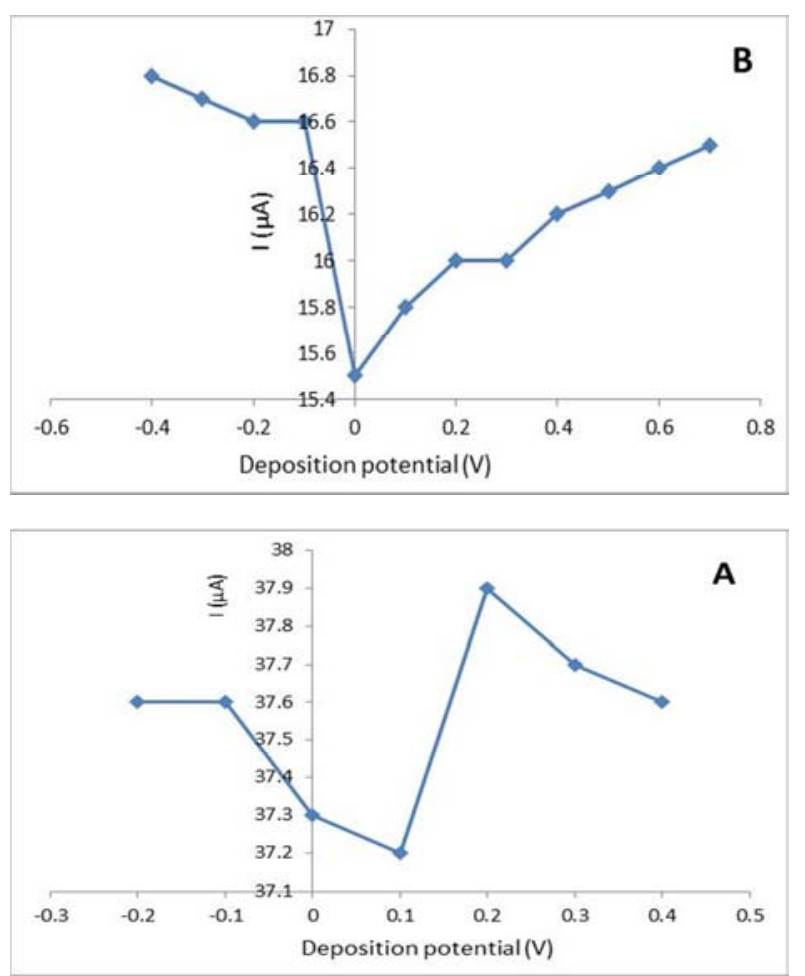

Figure 6. Effect of deposition potential (E) on the peak current of Mep in $0.04 M$ BR buffer $p H 6.0$ at (A) PGE and (B) GCE.

\section{Effect of deposition time}

The dependence of peak current developed in B-R buffer at the selected $\mathrm{pH}$ on the deposition time was also investigated (Figure 7) for an appropriate amount of Mep solution introduced to the electrolytic cell. From the plot it is clear that a short deposition time of the drug resulted in large anodic peak current. A full surface coverage was established after a certain accumulation time, 20 and 50 seconds are the selected deposition time at PGE and GCE, respectively.

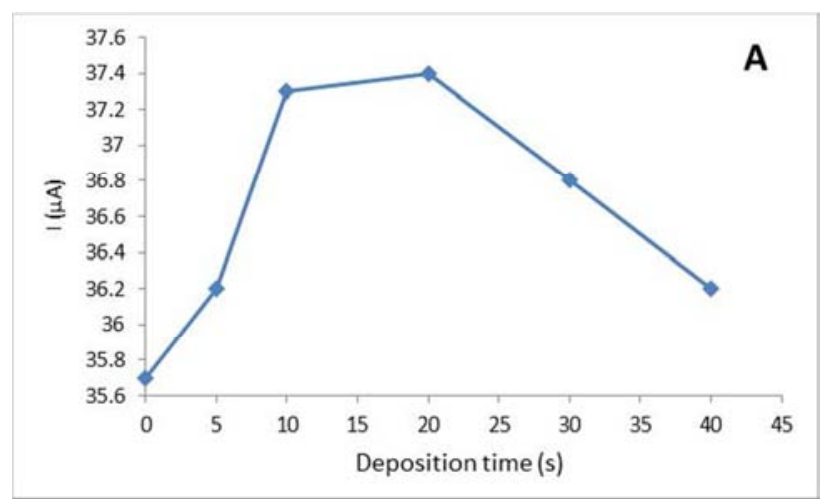




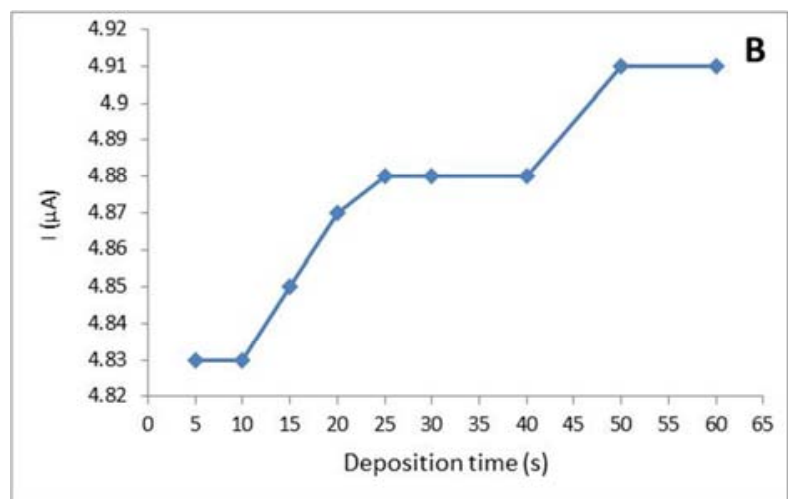

Figure 7. Effect of deposition time on the peak current of Mep in 0.04 M BR buffer $p H 6.0$ at (A) PGE and (B) GCE.

\subsection{Determination of Mep in Pure Form}

In order to develop a voltammetric method for determining Mep, we selected DPV and SWV methods, since the peaks are sharper and better-defined at lower concentration than those obtained by $\mathrm{CV}$ [37], in $0.04 \mathrm{M}$ BR buffer $\mathrm{pH} 6$ at both PGE and GCE, calibration graphs of the peak current I $(\mu \mathrm{A})$ against the drug concentration $(\mu \mathrm{g} / \mathrm{mL})$ were constructed (Figures 8 \& 9) [38], statistical parameters were calculated and shown in Table 1.
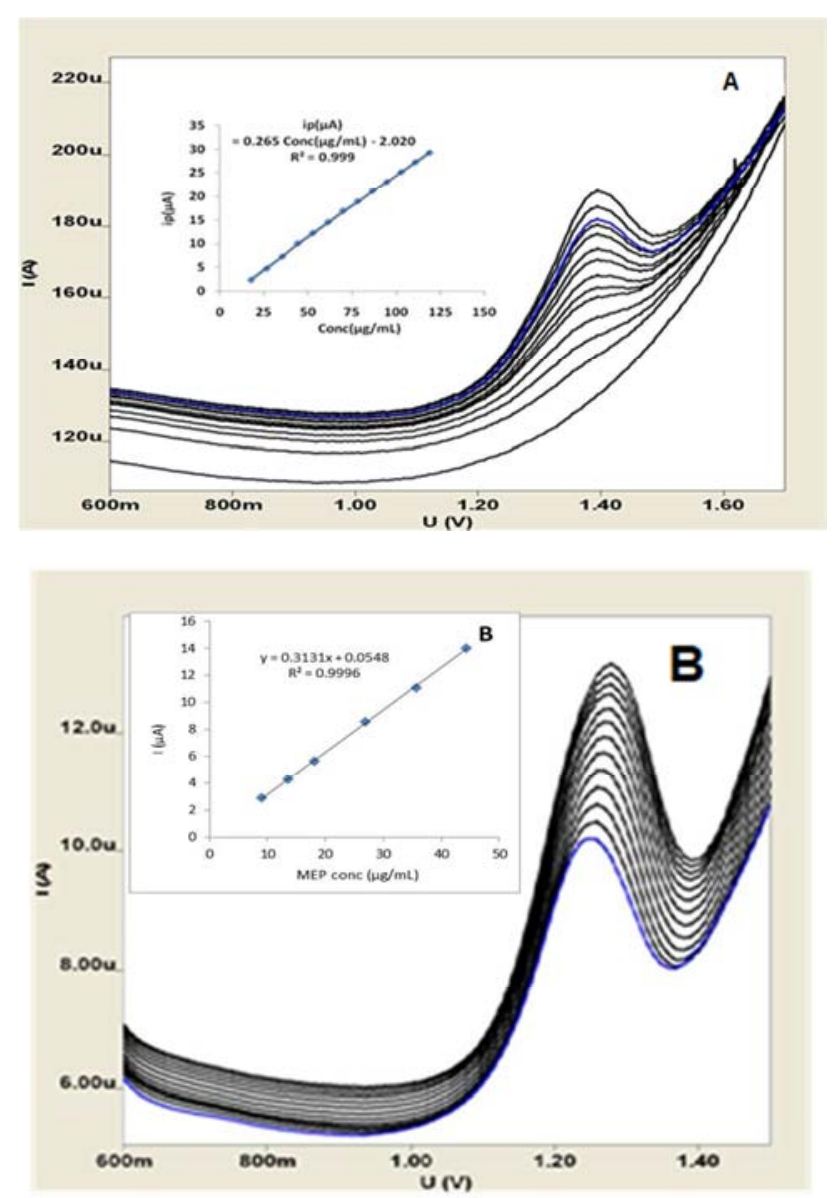

Figure 8. Differential pulse voltammograms responding for successive additions of Mep in $0.04 \mathrm{M}$ BR buffer pH 6.0 at (A) PGE and (B) GCE, the inset shows the corresponding calibration curves.
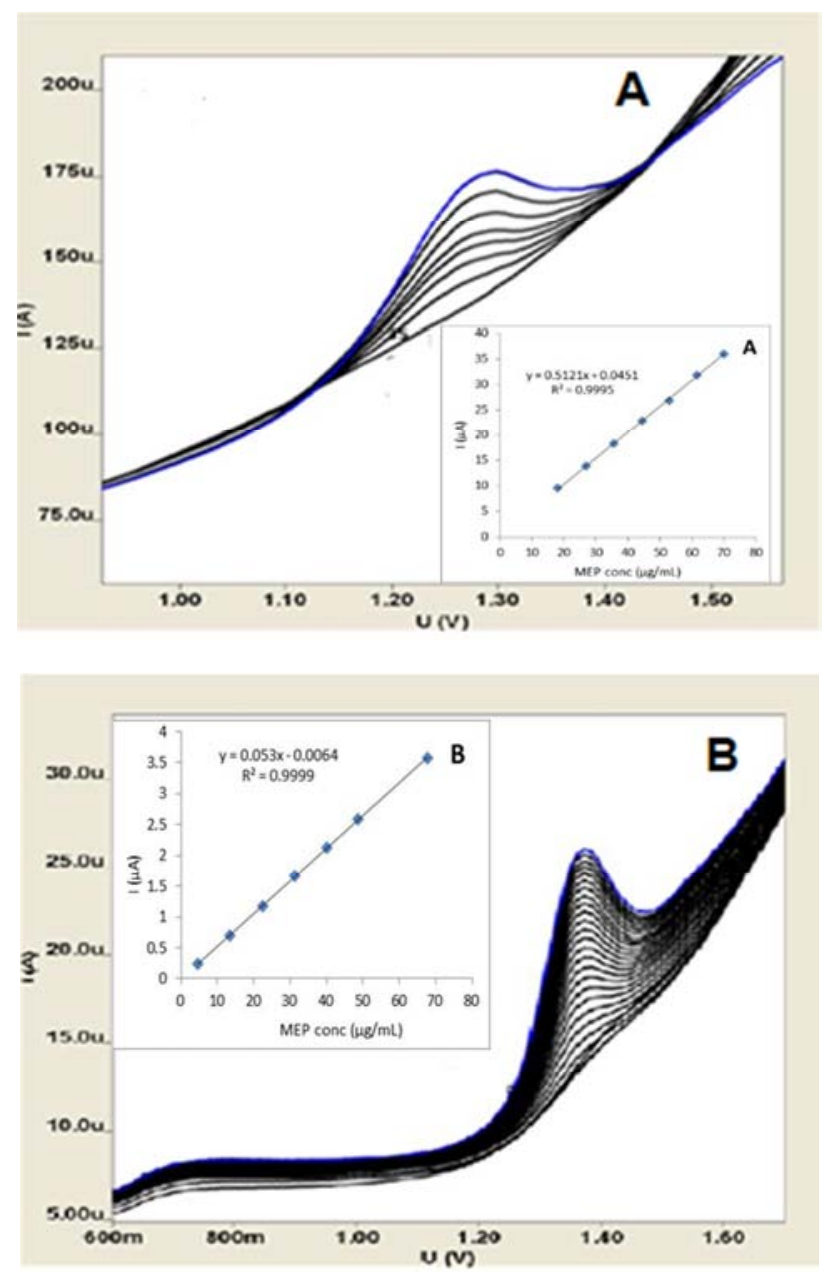

Figure 9. Square wave voltammograms responding for successive additions of Mep in 0.04 M BR buffer pH 6.0 at (A) PGE and (B) GCE, the inset shows the corresponding calibration curves.

\subsection{Determination of Mep in Pharmaceutical Dosage Form}

Determination of Mep in Decontractyl ${ }^{\circledR}$ tablets was accomplished as mentioned under (2.3.2) using the dosage form preparation. The experiment was repeated applying the standard addition technique, the corresponding regression equations were used to calculate the recovered concentrations of the labeled and the added concentrations of Mep. Statistical parameters were calculated and shown in (Table 1).

\subsection{Determination of Mep in both Spiked Human Mother Milk and Urine Samples}

The best results were obtained using human mother milk and urine samples replacing $10.0 \%$ of the buffer. The measurement of Mep in milk and urine samples was performed directly. The applicability of the SWV for the analysis of human milk and urine samples was obtained in (Figures $10 \& 11$ ), statistical parameters are shown in (Table 2). 

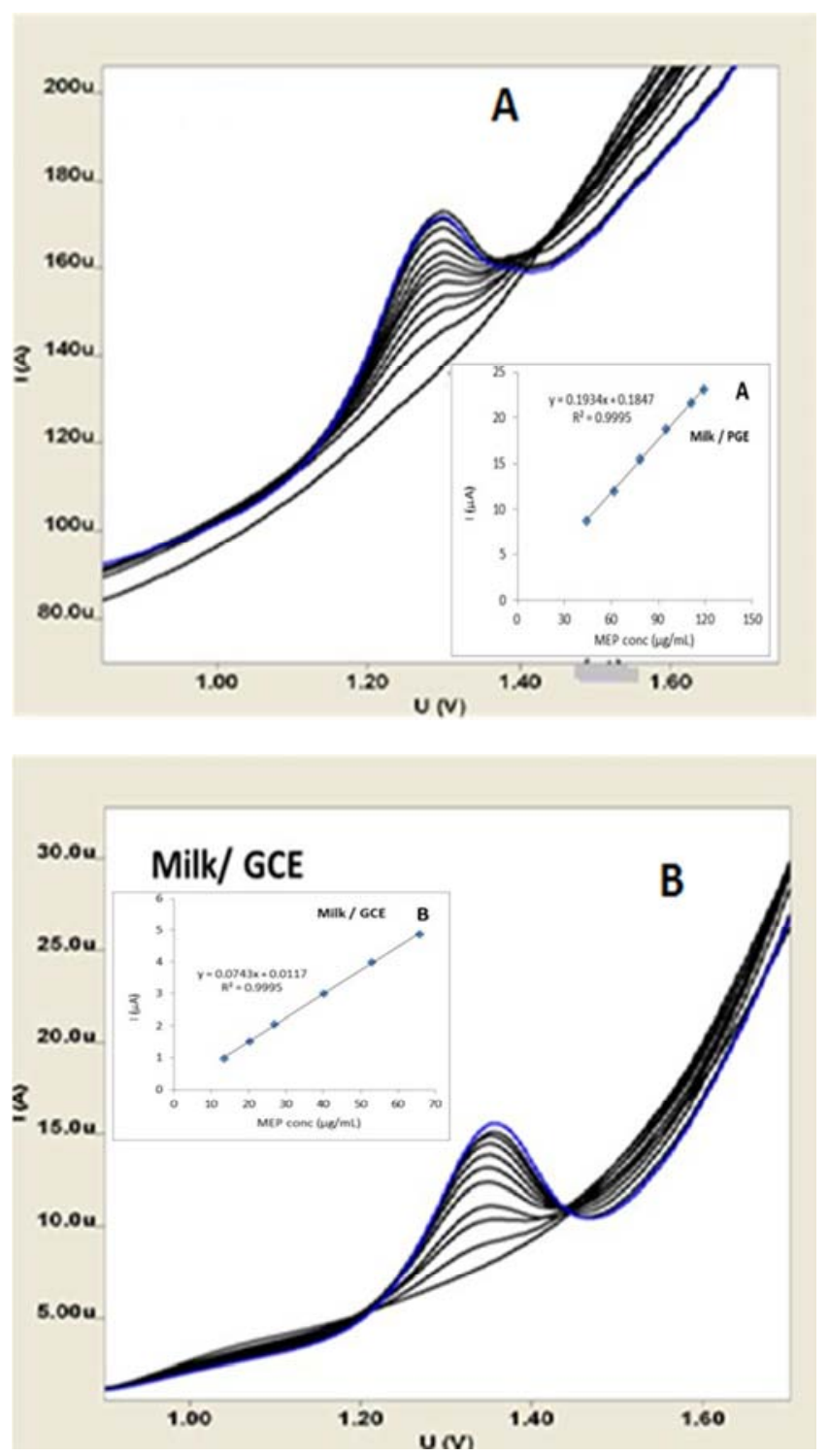

Figure 10. Square wave voltammograms responding for successive additions of Mep in spiked human mother milk sample at the optimum conditions for each electrode, the inset shows the corresponding calibration curves at (A) $P G E$ and (B) GCE.

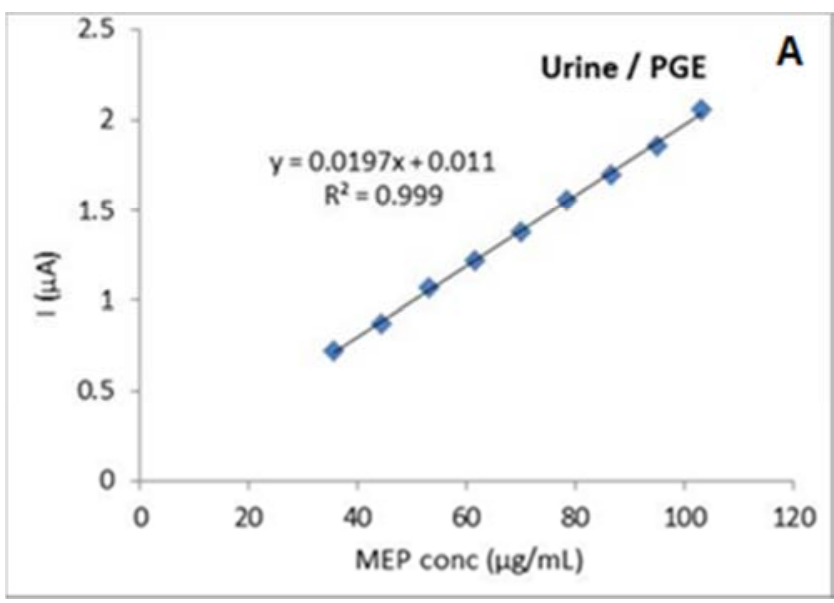

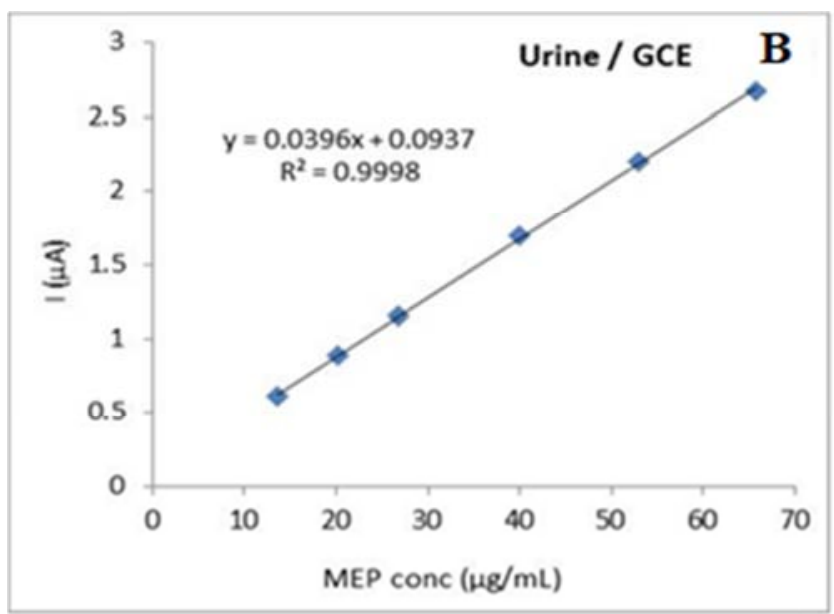

Figure 11. Calibration curves of Mep at optimum conditions for each electrode (A) PGE and (B) GCE in spiked human urine samples.

Analysis of drugs from biological samples usually requires extensive time consuming, sample preparation, use of expensive organic solvents and in sometimes use of other chemicals. In this study using SWV, no sample pretreatment was required. SWV was the method of choice due to its higher sensitivity, sharper and narrower peaks with good symmetrical pattern that made it preferred than CV.

Furthermore, no extra noise peaks were present in the biological peak occurred in the potential range where the analytical peak appeared. Stability of the milk and urine samples kept in refrigerator $\left(+4^{\circ} \mathrm{C}\right)$ was tested by making three consecutive analyses of the samples over a period of approximately 5 hours. There was no significant change in the peak currents and potentials between the first and last measurements.

\subsection{Effective Area of the Electrodes}

The effective area of the electrodes were calculated by the cyclic voltammetric method using $1.0 \mathrm{mM} \mathrm{K}_{4} \mathrm{Fe}(\mathrm{CN})_{6}$ at different scan rates. For a reversible process the following Randles- Sevcik formula (equation 8 ) was used:

$$
\text { Ipa }=\left(2.69 \times 10^{5}\right) \mathrm{A}_{\mathrm{o}} \mathrm{n}^{3 / 2} \mathrm{D}_{\mathrm{o}}^{1 / 2} \mathrm{C}_{\mathrm{o}} \mathrm{v}^{1 / 2}
$$

Where Ipa refers to the anodic peak current, $\mathrm{n}$ is the numbers of electrons transferred, $A_{o}$ is the surface area of the electrode, $D_{0}$ is the diffusion coefficient, $v$ is the scan rate and $\mathrm{C}_{0}$ is the concentration of $\mathrm{K}_{4} \mathrm{Fe}(\mathrm{CN})_{6}$. For $1.0 \mathrm{mM}$ $\mathrm{K}_{4} \mathrm{Fe}(\mathrm{CN})_{6}$ in $0.1 \mathrm{M} \mathrm{KCL}$ electrolyte, $\mathrm{n}=1, \mathrm{D}_{\mathrm{o}}=7.6 \times 10^{-6}$ $\mathrm{Cm}^{2} \cdot \mathrm{s}^{-1}$ then from the slope of the plot of Ipa versus $\mathrm{v}^{1 / 2}$, the electroactive surface area was calculated. In our experiment, the electroactive area of PGE and GCE were found to be 0.272 and $0.098 \mathrm{Cm}^{2}$, respectively. It is noticeable that PGE has greater active area compared to GCE, so there is a greater response of PGE than GCE on peak current that resulted from Mep drug. 


\section{Methods Validation}

\subsection{Linearity}

Calibration graphs from the standard solution of Mep according to the procedures described above were constructed by using DPV and SWV methods at both PGE and GCE for pure form and biological samples. Linear relationships in the concentration range are shown in (Figures $8 \& 9$, Table 1) for pure form, in (Figures 10\&11, Table 2) for human mother milk and urine. High values of square correlation coefficient $\left(\mathrm{R}^{2}\right)$ and small values of standard deviation (SD) and relative standard deviation (RSD) figure out the low scattering of the points around the calibration graphs and prove linearity of the methods through the specified concentration ranges. Above these ranges, loss of linearity is probably due to the saturation of the electrode surface with the drug.

\subsection{Limit of Detection (LOD) and Limit of Quantification (LOQ)}

The limits of detection (LOD) were determined by evaluating the lowest amount of analytes which can be detected but not necessarily quantitated as exact values, limits of quantification (LOQ) were determined by evaluating the lowest amount of analytes which can be quantitatively determined with suitable precision and accuracy in bulk and biological fluids (Tables $1 \& 2$ ).
These values are calculated on the peak current using the following equations as specified by ICH guidelines [40]:

$$
\mathrm{LOD}=3 \mathrm{~s} / \mathrm{m} \text { LOQ }=10 \mathrm{~s} / \mathrm{m}
$$

Where (s) is the standard deviation of the response and (m) is the slope of the calibration curve.

\subsection{Precision (Repeatability and Reproducibility)}

The repeatability (intra-day) and reproducibility (inter-day) of Mep were evaluated by assaying freshly prepared solutions in triplicates on the same day and on three different days using the proposed procedures, respectively. The RSD\% indicated high accuracy and precision and proved to be suitable for quality control of Mep (Tables $1 \& 2$ ).

\subsection{Applicability}

The proposed methods were successfully applied for the determination of Mep in pharmaceutical dosage form and biological fluids (human mother milk and urine). The obtained results showed good accuracy with acceptable standard deviation and with the application of standard addition technique in dosage form and very low values of LOD and LOQ in human biological fluids that help the earlier detection and quantitation of Mep preventing its poisoning and fatal effects (Tables $1 \& 2$ ).

Table 1. Validation parameters and results obtained by the proposed DPV and SWV methods at PGE and GCE for the determination of Mep in bulk and dosage form.

\begin{tabular}{|c|c|c|c|c|}
\hline \multirow{2}{*}{$\begin{array}{l}\text { Electrode/method } \\
\text { Parameter }\end{array}$} & \multicolumn{2}{|l|}{ PGE } & \multicolumn{2}{|l|}{ GCE } \\
\hline & DPV & SWV & DPV & SWV \\
\hline Potential value E (V) & 1.38 & 1.28 & 1.3 & 1.39 \\
\hline Maximum pH & \multicolumn{2}{|l|}{6.0} & \multicolumn{2}{|l|}{6.0} \\
\hline Deposition potential (E) & \multicolumn{2}{|l|}{0.2} & \multicolumn{2}{|l|}{-0.4} \\
\hline Deposition time (s) & \multicolumn{2}{|l|}{20} & \multicolumn{2}{|l|}{50} \\
\hline Electrochemical process behavior & \multicolumn{2}{|l|}{ Adsorption } & \multicolumn{2}{|c|}{ Adsorption with some diffusion } \\
\hline Linear range conc $(\mu \mathrm{g} / \mathrm{mL})$ & $18.02-119.07$ & $18.02-70.0$ & $9.05-44.39$ & $4.54-65.78$ \\
\hline Regression coefficient $\left(\mathrm{R}^{2}\right)$ & 0.9997 & 0.9995 & 0.9995 & 0.9999 \\
\hline $\mathrm{LOD}(\mu \mathrm{g} / \mathrm{mL})$ & 0.13 & 0.06 & 0.61 & 0.95 \\
\hline LOQ $(\mu \mathrm{g} / \mathrm{mL})$ & 0.39 & 0.18 & 1.84 & 2.88 \\
\hline Intra-day (R.S.D\%) & $0.06-0.17$ & $0.12-0.55$ & $0.29-0.64$ & $0.34-0.98$ \\
\hline Inter-day (R.S.D\%) & $1.18-1.64$ & $0.81-1.78$ & $1.30-1.99$ & $1.12-1.96$ \\
\hline Accuracy (Mean Drug in bulk & $100.58 \% \pm 1.15$ & $99.76 \% \pm 1.16$ & $100.17 \% \pm 1.14$ & $100.59 \% \pm 1.18$ \\
\hline recovery $\% \pm \quad$ Drug in dosage form & $99.93 \% \pm 1.52$ & ---- & ---- & $100.57 \% \pm 1.24$ \\
\hline
\end{tabular}

Table 2. Validation parameters and results obtained by the proposed SWV at PGE and GCE for the determination of Mep in presence of human mother milk and urine.

\begin{tabular}{|c|c|c|c|c|}
\hline Electrode & PGE & & GCE & \\
\hline Parameter & MILK & URINE & MILK & URINE \\
\hline Potential value E (V) & 1.28 & 1.3 & 1.34 & 1.36 \\
\hline Linear range $(\mu \mathrm{g} / \mathrm{mL})$ & $44.39-119.07$ & $35.69-103.02$ & $13.55-65.78$ & \\
\hline Regression equation & $\mathrm{I}=0.1934$ Conc +5.4847 & $\mathrm{I}=0.0197$ Conc +0.494 & $\mathrm{I}=0.0743$ Conc +0.3801 & $\mathrm{I}=0.0396$ Conc +0.3937 \\
\hline Regression coefficient $\left(\mathrm{R}^{2}\right)$ & 0.9995 & 0.9993 & 0.9995 & 0.9998 \\
\hline LOD $(\mu \mathrm{g} / \mathrm{mL})$ & 0.99 & 0.97 & 0.68 & 0.83 \\
\hline LOQ $(\mu \mathrm{g} / \mathrm{mL})$ & 2.99 & 2.93 & 2.06 & 2.53 \\
\hline Intra-day (R.S.D\%) & $0.19-0.77$ & $0.28-0.69$ & $0.24-0.66$ & $0.21-0.83$ \\
\hline Inter-day (R.S.D\%) & $1.28-1.94$ & $1.38-1.81$ & $0.82-1.28$ & $1.22-1.73$ \\
\hline Accuracy (Mean recovery $\% \pm \mathrm{SD}$ ) & $100.05 \% \pm 1.47$ & $99.78 \% \pm 0.58$ & $99.95 \% \pm 1.68$ & $98.58 \% \pm 1.60$ \\
\hline
\end{tabular}




\subsection{Robustness}

The robustness of an analytical procedure is a measure of its capacity to remain unaffected by small, but deliberate variations in method's parameters. In our work, the robustness of the proposed method was demonstrated by constancy of the peak current with deliberated minor changes in some experimental parameters, including the change in $\mathrm{pH}( \pm 0.2)$ at both PGE and GCE and deposition time, the time considered before each measurement ( $\pm 2 \mathrm{sec}$ at PGE) and ( $\pm 5 \mathrm{sec}$ at GCE). Only one factor was changed at a time, while the other was kept constant. These minor changes that may take place during the experimental operation did not affect the peak current intensity of the studied drug indicating the reliability of the proposed methods during normal usage (Table 3 ).

Table 3. The robustness of the proposed methods for the determination of Mep at PGE and GCE using SWV.

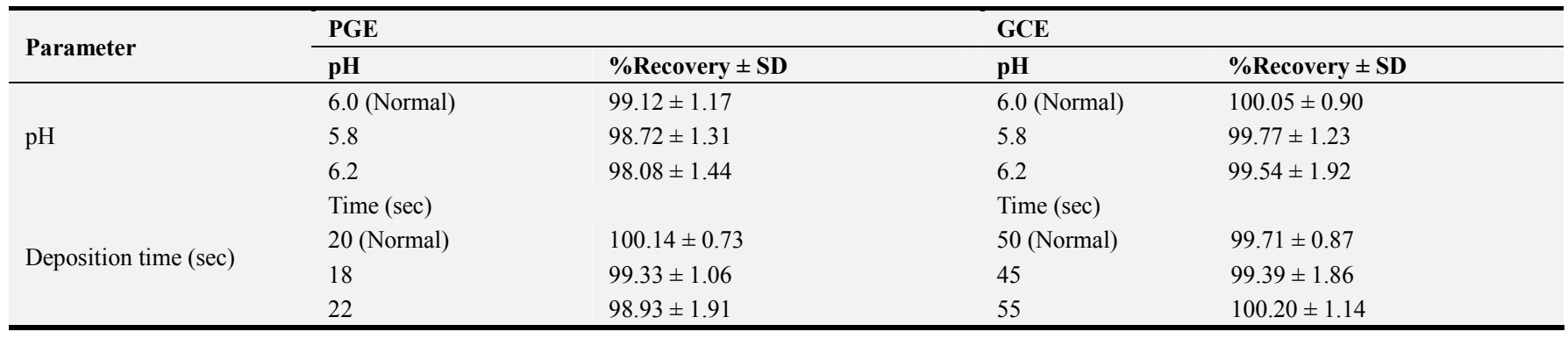

*The values in the table are mean of three determinations.

\subsection{Statistical Analysis}

Statistical analysis of the results obtained by the proposed methods for the determination of Mep and the official method was carried out by applying student's t-test and variance ratio $\mathrm{F}$-test at $\mathrm{P}=0.05$. The results showed that the student's t-test and variance ratio $F$-test at $\mathrm{P}=0.05$ are less than the tabulated ones as shown in table 4 . This ascertained that there is no significant difference among methods.

Table 4. Statistical comparison among the recovery results of Mep in its pure form using DPV and SWV methods at both PGE and GCE and the official method.

\begin{tabular}{lllll}
\hline \multirow{2}{*}{ Component } & DPV & & SWV & \\
\cline { 2 - 4 } & PGE & GCE & PGE & GCE \\
\hline Mean & 100.58 & 100.17 & 99.76 & 100.59 \\
SD & 1.15 & 1.14 & 1.16 & 1.18 \\
n & 5 & & & 0.89 .79 \\
Variance & 1.319 & 1.292 & 1.346 & 1.381 \\
*t-test (2.306) & 1.221 & 0.596 & 0.049 & 1.234 \\
*F-test (6.388) & 1.780 & 1.744 & 1.816 & 0.741 \\
\hline
\end{tabular}

*Figures in parenthesis are the theoretical $\mathrm{t}$ and $\mathrm{F}$ values at $\mathrm{p}=0.05$ while $\mathrm{n}$ is the number of determination.

**Official method for Mep is a titrimetric method (Indian Pharmacopoeia 1985).

\section{Conclusion}

Validated differential pulse and square wave voltammetric methods have been developed, validated and successfully applied to the estimation of Mep in pure form, pharmaceutical dosage form, human mother milk and urine samples at both PG and GC electrodes. PGE introduces several advantages over GCE constituting in being disposable, available, cheaper, more sensitive, moreover, the easiness of cleaning the surface. The proposed methods are direct, sensitive, inexpensive, simple, accurate, precise, more safe and do not need the elaborated treatment and tedious extractions required in other analytical methods. The main advantage of the methods is rapidity as they require less than five minutes to run a sample. Applicability of our new methods in the biological samples is the stone of the corner of our aim of this study to help in rapid detection and prevention of Mep intoxication that introduces preliminary vision for the clinical investigations.

\section{Acknowledgements}

This work was completely accomplished in the National Organization for Drug Control and Research (NODCAR), Giza, Egypt; by supplying all apparatus, chemicals and reagents.

\section{References}

[1] A. Brayfield., The complete drug reference, Martindale. $38^{\text {th }}$ ed. Vol. A. (2014), London: Pharmaceutical Press.

[2] G. Frederick Hofmann, S. Walter Root and M. Cedric Smith, The Nervous System: Central Nervous System Drugs, 2 (1965) 14-28.

[3] P. Guinebault, C. Colafranceschi and G. Bianchetti, Journal of Chromatography A, 507 (1990) 221-225. 
[4] S. V. Mulgund, M. S. Phoujdar, S. V. Londhe, P. S. Mallade, T S. Kulkami, A. S. Deshpande and K. S. Jain, Indian Journal of Pharmaceutical Sciences, 71(2009) 35-40.

[5] M. B. Devani, C. J. Shishoo, B. N. Suhagia and S. A. Shah, Indian Journal of Pharmaceutical Sciences, 56 (1994) 41-44.

[6] A. R. Maass, P. L. Carey and A. E. Heming, Journal of Analytical chemistry, 31 (1959) 1331-1334.

[7] A. M. Gillespie and S. M. Walters, Journal of Analytical letters, 6 (1973) 61-69.

[8] F. Sayin and S. Kir, Journal of Pharmaceutical Biomedical Analysis, 25 (2001) 153-163.

[9] S. A. Ö zkan, B. Uslu and P. uman, Analytical Chim. Acta., 457 (2002) 265-274.

[10] B. Uslu and S. A. Özkan, Electrochimica acta, 49 (2004) 43214329.

[11] J.-M. Kauffmann and J.-C. Vire, Analytica chimica acta, 273, $1-2$, (1993) 329-334.

[12] N. J. Ronkainen, H. B. Halsall and W. R. Heineman, Chemical Society Reviews, 39(2010) 1747-1763.

[13] J. Wang, John Wiley \& Sons, 1988.

[14] S. A. Özkan, B. Uslu and Z. Sentürk, Electroanalysis, 16, 3 (2004) 231-237.

[15] M. A. Koda-Kimble and L. Y. Young, Applied Therapeutics: Clinical Use of Drugs vol. 1, $5^{\text {th }}$ ed., (1992) 18-5.

[16] R. Ouyang, Z. Zhu, C. E. Tatum, J. Q. Chambers and Z.-L. Xue, Journal of Electroanalytical Chemistry, 656 (2011) 78-84.

[17] E. Dede, Ö. Saglam and Y. Dilgin, Electrochimica Acta, 127(2014) 20-26.

[18] A. Levent, Y. Yardim and Z. Senturk, Electrochimica Acta, 55, 1(2009)190-195.

[19] W. Gao, J. Song, and N. Wu, Journal of Electroanalytical Chemistry, 576, 1(2005)1-7.

[20] D. Demetriades, A. Economou, and A. Voulgaropoulos, Analytica Chimica Acta, 519, 2(2004) 167-172.

[21] H. Karadeniz, B. Gulmez, F. Sahinci et al, Journal of Pharmaceutical and Biomedical Analysis, 33, 2 (2003) 295302.

[22] A. M. Bond, P. J. Mahon, J. Schiewe, and V. Vicente-Beckett, Analytical Chemistry Acta, 345, 1-3, (1997) 67-74.
[23] M. Rizk, H. A. Hendawy, M. M. A. El-Alamin and M. I. Moawad, Journal of Electroanalytical Chemistry, 749 (2015) 53-61.

[24] M. Rizk, M. M. Abou El-Alamin, H. A. Hendawy and M. I. Moawad, Electroanalysis, (2015).

[25] H. T. S. Britton and R. A. Robinson, Journal of the Chemical Society (Resumed), (1931) 1456-1462.

[26] J. Heyrovsky and P. Zuman, Practical Polarography, Academic Press, New York, (1968) 163-179.

[27] S. Shen, H. Shi and H. Sun, International Journal of Chemical Kinetics, 39 (2007) 440-446.

[28] E. Laviron, Journal of Electroanalytical Chemistry, 101(1979), 19-28.

[29] H. Elqudaby, G. G. Mohamed and G. M. El Din, International Journal of Electrochemical Sciences, 9 (2014) 856-869.

[30] A. Radi and Z. El-Sherif, Talanta 58 (2002) 319-324.

[31] B. Dogan-Topal, B. Bozal-Palabıyık, B. Uslu and S. A. Ozkan, Sensors Actuators B Chemical, 177 (2013) 841-847.

[32] A. Shalaby, W. S. Hassan, H. A. Hendawy and A. Ibrahim, Journal of Electroanalytical Chemistry, 763 (2016) 51-62.

[33] M. A. El-ries, G. G. Mohamed and A. K. Attia, YAKUGAKU ZASSHI, the pharmaceutical society of Japan, 128(2008) 171177.

[34] D. K. Gosser, Cyclic Voltammetry: Simulation and Analysis of Reaction Mechanisms, VCH, New York, N. Y., 1993.

[35] A. J. Bard and L. R. Faulkner, Elecrochemical Techniques Fundamentals and Applications, second ed. John Wiley and Sons, New York, 2001.

[36] L. Agü1, A. Guzmán, P. Yáñez-Sedeño and J. Pingarrón, Analytica Chimica Acta, 461 (2002) 65-73.

[37] C. M. Riley and T. W. Rosanske, Development and validation of analytical techniques, Elsevier, 1996.

[38] B. Uslu and S. A. Özkan, Analytica Chimica Acta, 462 (2002) 49-57.

[39] B. Dogan-Topal, B. Uslu and S. A. Özkan, Sensors and Actuators B: Chemical, 177 (2013) 841-847.

[40] M. E. Swartz and I. S. Krull, Handbook of analytical validation, CRC Press, 2012. 